\title{
Localization of Adherens Junction Proteins along the Possible Sliding Interface between Secretory Ameloblasts of The Rat Incisor
}

\author{
Sumio Nishikawa1), Sachiko Tsukita*, Shoichiro Tsukita* and Shozo Sasa \\ Department of Histology, Kanagawa Dental College, Yokosuka, Kanagawa Dental College, Yokosuka, \\ Kanagawa 238, and ${ }^{*}$ Department of Ultrastructural Research, The Tokyo Metropolitan Institute of Medical \\ Science, Bunkyo-ku, Tokyo 113, Japan
}

Key words: adherens junction/radixin/vinculin/cell motility/ameloblast

\begin{abstract}
$A B S T R A C T$. Localization of junctions between inner enamel-secretory ameloblasts was examined by immunofluorescence microscopy using antibodies against adherens junction proteins, radixin, vinculin, and A-CAM. All antibodies used stained the boundary between the ameloblasts exclusively in the plane where $F$-actin was abundant. This suggests that the adherens junctions in the ameloblasts are involved in cell-to-cell movement with actin-based microfilament bundles.
\end{abstract}

Ameloblasts, which are responsible for the formation of tooth enamel, have junctional complexes at both their apical and distal poles during the secretory stage $(12,17)$. There are two secretory stages of enamel formation (19). The first stage, inner enamel secretion, occurs when ameloblasts produce rows and the secretory processes (Tomes' processes) of the ameloblasts take on an opposite orientation between adjacent rows of ameloblasts (5). The inner enamel is composed of rows of rods which are structural units of enamel, and the long axis of th rods within a row runs almost at right angles to that of the adjacent row (18). The second stage, outer enamel secretion, occurs when ameloblasts lose their row pattern and, the rods formed become uniformly straight $(5,18,19)$. Studies on the structure of the inner enamel suggest that the ameloblasts slide laterally past each other during inner enamel formation and that the microfilament bundles associated with the distal junctional complexes are responsible for cellcell sliding between the rows of ameloblasts $(5,7-10)$. It has also been shown that the microfilament bundles in the ameloblasts are composed of actin, myosin, alphaactinin and tropomyosin (8). These cytoskeletal proteins are abundant at the cell boundary in the plane where cell-cell sliding takes place. Therefore, it is reasonable to assume that the contraction of actinbased filament bundles causes the ameloblast rows to slide laterally. As to how contraction of the filament bundles in the inner enamel-secretory ameloblasts leads

1) Present address: Department of Biology, Tsurumi University, School of Dental Medicine, 2-1-3 Tsurumi, Tsurumi-ku, Yokohama 230, Japan. to actual cell movement, to produce actual sliding between adjacent cells some transient fixing points must be present in the sliding plane with the contraction of the filament bundles. In this study, we concentrated on adherens junction proteins, since adherens junctions are known to be highly dynamic structures as well as anchoring loci between neighboring cells or cells and substrates.

Three kinds of proteins were examined by immunofluorescence microscopy: radixin, an adherens junction protein located at the cell-cell junction but not at the cell-substrate junction (13) vinculin, another adherens junction protein located at both the cell-cell and cellsubstrate junctions $(2,3)$; and A-CAM, a $\mathrm{Ca}^{2+}$-dependent cell adhesion molecule, located at the adherens junctions $(15,16)$. In this study, all three proteins were localized in the distal junctional area, and were exclusively at the cell boundary along the possible sliding plane of the inner enamel-secretory ameloblasts.

\section{MATERIALS AND METHODS}

Male Wistar rats (130-310 g) were sacrificed by decapitation under sodium pentobarbital anesthesia. Their mandibular incisors were cut with a razor blade 3 to $4 \mathrm{~mm}$ from the apical end. The apical tips were frozen by dipping them into liquid nitrogen, and cryosections $(6-8 \mu \mathrm{m})$ were made using a cryotome (Tissue Tek II, Miles IL/USA). The sections were fixed on glass microscope slides with $95 \%$ ethanol at $-20^{\circ} \mathrm{C}$ for $30 \mathrm{~min}$, and then immersed in 100\% acetone at room temperature for $5 \mathrm{~min}$. The antibodies used in this study included polyclonal rabbit antibody against rat liver radixin (13), polyclonal rabbit antibody against rat liver vinculin $(13,14)$, and 
monoclonal mouse antibody against A-CAM (Sigma, MO/USA). The fixed sections were first labeled for $30 \mathrm{~min}$ with antiradixin diluted $1: 100$ with $1 \%$ bovine serum albumin (BSA) in phosphate buffered saline (PBS), with antivinculin diluted $1: 50$ with $1 \%$ BSA in PBS, or with anti-ACAM diluted $1: 50$ with $1 \%$ BSA in PBS. Control sections were incubated with normal rabbit serum (Cappel, PA/USA) or control ascites fluid (ICN, IL/USA) instead of the specific primary antibodies. Next, they were labeled for $30 \mathrm{~min}$ with FITC-conjugated anti-rabbit Ig (Amersham, Bucks./UK) diluted $1: 20$ with 1\% BSA in PBS or FITC-conjugated antimouse Ig (Amersham) diluted $1: 20$ with $1 \%$ BSA in PBS. After being whashed with PBS three times for $5 \mathrm{~min}$ each, the stained sections were mounted in Perma Fluor (Lipshaw Immunon, MI/USA). Some sections stained with antiradixin or antivinculin were further labeled with rhodamine-phalloidin (Molecular Probes, OR/USA) for localization of F-actin. The sections were examined with an Olympus microscope equipped with epifluorescence optics (Olympus, Tokyo/Japan). Double-labeled sections were examined at $B$ excitation using a G-520 filter (Olympus) to block rhodamine fluorescence Micrographs were taken using Kodak Tri-X pan film (Eastman Kodak NY/USA).

\section{RESULTS}

Antibodies against radixin, vinculin and A-CAM labeled the junctional areas associated with the microfilament bundles in the inner enamel-secretory ameloblasts (Figs. 1, 3 and 8). Oblique transverse sections of the ameloblasts enabled us to visualize the polarized pattern of actin-based filament bundles and associated junctions in the distal pole. Dense microfilament bundles revealed by rhodamine-phalloidin staining were localized circumferentially at the periphery of cross-sectioned rectanglar cell profiles at the level of the distal junctional area. The microfilament bundles were localized at the peripheral cytoplasm corresponding to the two sides of the rectangle but they were often absent in the other plane (Figs. 6b and 7b). All antibodies stained the cell boundary corresponding to the same two sides of the rectangles as the microfilament-rich sides (Figs. 6, 7 and 8). antiradixin and antivinculin stained the distal junctional area intensely, while anti-A-CAM stained the cell boundary as thin rods or dots (Figs. 6, 7 and 8). Antiradixin and antivinculin also stained the proximal junctional area as a group of bright rings (Figs. 4 and 5). Secretory ameloblasts release enamel matrix proteins from the distal end in a process called Tomes' process. The Tomes' processes were labeled with antivinculin but not with antiradixin (Figs. 6a and 7a).

Odontoblast junctional complexes, which were located just beneath the predentin and associated with microfilament bundles, were labeled with both antiradixin and antivinculin (Figs. 2 and 3). In addition, antiradixin labeled the blood capillaries in the dental pulp and in the connective tissue adjacent to the enamel organ (Figs. 1, 2 and 4). The control sections incubated with normal rabbit serum or control ascites fluid did not label these odontogenic tissues.

Fig. 1. Fluorescence micrograph of inner enamel-secretory ameloblasts stained with antiradixin (a) and corresponding phase-contrast micrograph (b). proximal (p) and distal (d) junctional areas and blood capillary (arrows) are stained. A, ameloblasts; E, enamel. Bars $=20 \mu \mathrm{m}$.

Fig. 2. Fluorescence micrograph of odontoblasts facing the inner enamel-secretory ameloblasts stained with antiradixin. The junctional areas of the odontoblasts are stained in (a) (arrowheads), and corresponding phase-contrast micrograph is shown in (b). A blood capillary in the odontoblast layer is stained (an arrow). $\mathrm{O}$, odontoblasts. Bars $=20 \mu \mathrm{m}$.

Fig. 3. Fluorescence micrographs of inner enamel-secretory ameloblasts and odontoblasts stained with antivinculin (a) and rhodaminephalloidin for F-actin (b). Corresponding phase-contrast micrograph is shown in (c). Proximal and distal (d) junctional areas and the Tomes' processes (arrows) of the junctional area (arrowheads) are stained with antivinculin and rhodamine-phalloidin. A, ameloblsats; E, enamel; D, dentin; O, odontoblasts. Bars $=20 \mu \mathrm{m}$.

Fig. 4. An oblique cross-section of inner enamel-secretory ameloblasts and the overlying epithelial and connective tissues stained with antiradixin. A group of uniform polygons $(p)$ in the proximal junctional area of the ameloblasts is seen. Blood capillaries are stained (arrows). $\mathrm{Bar}=20 \mu \mathrm{m}$.

Fig. 5. An oblique cross-section of inner enamel-secretory ameloblasts stained with antivinculin. A group of uniform polygons (p) in the proximal junctional area of the ameloblasts is seen. Bar $=20 \mu \mathrm{m}$.

Fig. 6. An oblique cross-section of the distal poles of inner enamel-secretory ameloblasts doubly stained with antiradixin (a) and rhodaminephalloidin (b). A higher magnification of (a) is shown in (c). Cross-sectioned cell profiles of the distal junctional area (d) are rectangular. Both antiradixin (a) and rhodamine-phalloidin (b) are exclusively localized along the probable sliding plane (orientation is indicated by an arrow) and are only faintly seen in the orthogonal plane. Tomes' processes $(\mathrm{t})$ are intensely stained with rhodamine-phalloidin (c), but not with antiradixin (a). At a higher magnification (c), cross-sectioned cell profiles at the level of the distal junctional area are aligned in rows along the probable sliding plane. The cell profiles making a row are indicated by (r). Intense stainings of antiradixin along the probable sliding plane (indicated by an arrow in c) make parallel lines. (a, b) Bars $=10 \mu \mathrm{m}$. (c) Bar $=2 \mu \mathrm{m}$.

Fig. 7. An oblique cross-section of the distal poles of inner enamel-secretory ameloblasts stained with antivinculin (a) and rhodaminephalloidin (b) and the corresponding phase-contrast micrograph (c). Both antivinculin and rhodamine-phalloidin are colocalized at the cell periphery of the distal junctional area (d) and Tomes' processes ( $t$ ). In the distal junctional area, both antivinculin and rhodamine-phalloidin are localized at the cell boundary along the probable sliding plane (orientation of the sliding plane is indicated by an arrow). Bars $=10 \mu \mathrm{m}$.

Fig. 8. An oblique cross-section of the distal junctional area of inner enamel-secretory ameloblasts stained with anti-A-CAM. Stained rods or dots (arrowheads) are located at the cell boundary along the probable sliding plane (orientation of the sliding plane is indicated by an arrow). $\mathrm{Bar}=10 \mu \mathrm{m}$. 

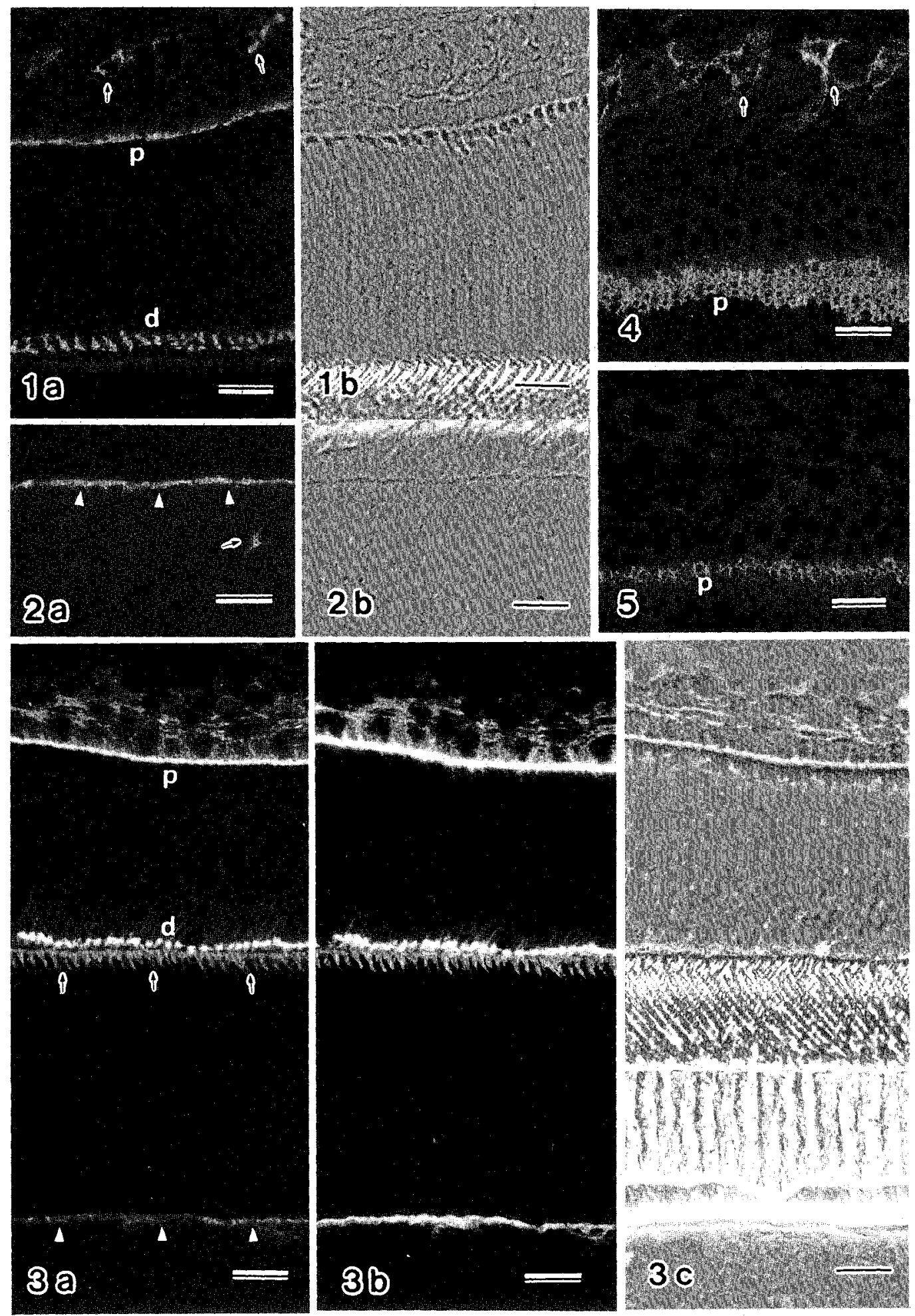

Figs. 1-5 
S. Nishikawa et al.

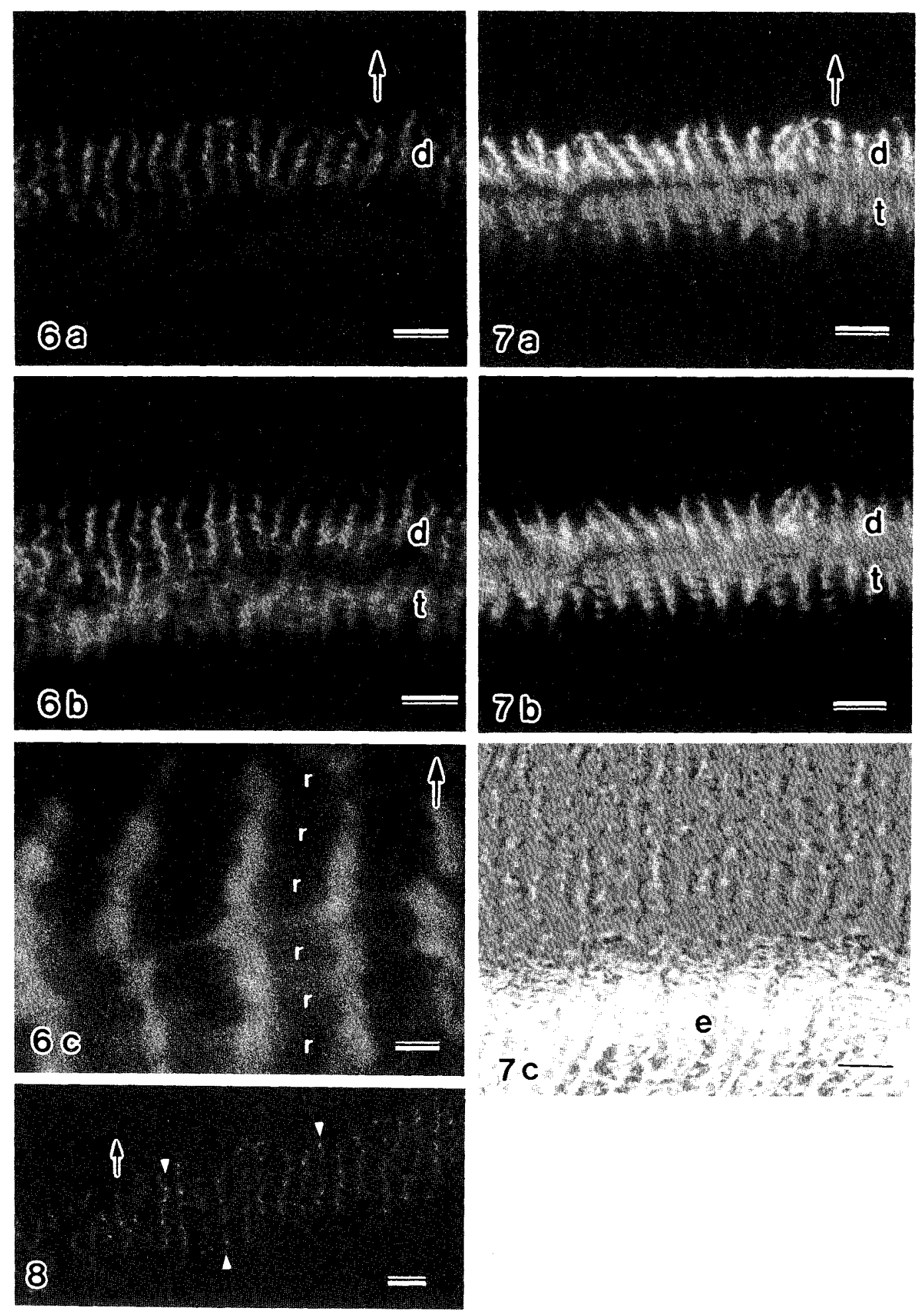

Figs. 6-8 


\section{DISCUSSION}

A previous study showed that an antibody against chicken gizzard vinculin labeled the distal poles of inner enamel-secretory ameloblasts (8). In this study using well characterized antibody against rat liver vinculin $(13,14)$, the proximal and distal junctional area as well as the Tomes' processes were clearly labeled. This apparent discrepancy may reflect differences in the accessibility of the two kinds of antibodies to rat ameloblast vinculin.

Radixin failed to label Tomes' processes when cellsubstrate junctions were present. The results of this study are consistent with those of previous reports that indicate that vinculin is present in both the cell-cell and cell-substrate junctions, and radixin present only at cell-cell junctions (13).

This study revealed that, in inner enamel-secretory ameloblasts, there are three kinds of adherens junction proteins located at the cell boundary in the plane where cell-cell sliding may occur. A previous study showed that antibody against desmoplakin I/II stained the cell boundaries of the distal junctional area of ameloblasts exclusively in a plane where cell-cell sliding may occur (8), suggesting that the desmosomes were preferentially localized in the sliding plane. We have shown that actinbased microfilament bundles which contain myosin, alpha-actinin and tropomyosin, run along this plane (8). Although the contraction of the microfilament bundles certainly generates a force for active sliding, some other mechanisms such as transient fixing points are necessary to produce relative translocation between two adjacent ameloblasts. It has been shown that the adherens junctions and desmosomes are highly dynamic structures (4). For regulation of junctional stability, the presence of proteins with kinase activity $(6,11)$ or protease activity (1) in cell-substrate adherens junctions has been suggested. The preferential presence of adherens junction proteins and desmosome protein at the interface of cell-cell sliding suggests that both junctions are involved in active ameloblast movement and the formation of the unique rod arrangement.

\section{REFERENCES}

1. Beckerle, M.C., Burridge, K., DeMatino, G.N., and Croall, D.E. (1987). Colocalization of clacium-dependent protease II and one of its substrates at sites of cell adhesion. Cell 51: 569-577.

2. GeIGER, B. (1979). A $130 \mathrm{k}$ proteins from chicken gizzard: Its localization at the termini of microfilament bundles in cultured chicken cells. Cell 18: 193-205.

3. Geiger, B., Dutton, A.H., Tokuyasu, K.T., and Singer, S.J. (1981). Immunoelectron microscope studies of membranemicrofilament interaction. The distribution of $\alpha$-actinin, tropomyosin and vinculin in intestinal epithelial brush border and chicken gizzard smooth muscle cells. J. Cell. Biol. 91: 614628.

4. Green, K.J., Geiger, B., Jones, J.C.R., Talian, J.C., and GoLdman, R.D. (1987). The relationship between intermediate filaments and microfilaments before and during the formation of desmosomes and adherens-type junctions in mouse epidermal keratinocytes. J. Cell. Biol. 104: 1389-1402.

5. Kallenbach, E. (1973). The fine structure of Tomes' process of rat incisor ameloblasts and its relationship to the elaboration of enamel. Tissue Cell 5; 501-524.

6. Kellie, S., Patel, B., Wigglesworth, N.M., Critchley, D.R., and WYKE, J.A. (1986). The use of Rous sarcoma virus transformation mutants with differing tyrosine kinase activities to study the relationship between vinculin phosphorylation, pp $60^{\mathrm{v}-\mathrm{src}}$ location and adhesion plaque integrity. Exp. Cell. Res. 165: 216-228.

7. NishiKawa, S. and Kitamura, H. (1986). Localization of actin during differentiation of the ameloblast, its related epithelial cells and odontoblasts in the rat incisor using NBD-phallacidin. Differentiation 30: 237-243.

8. Nishikawa, S., Fujiwara, K., and Kitamura, H. (1988). Formation of the tooth enamel rod pattern and the cytoskeletal organization in secretory ameloblasts of the rat incisor. Eur. J. Cell. Biol. 47: 222-232.

9. ReITH, E.J. and Ross, M.H. (1973). Morphological evidence for the presence of contractile elements in secretory ameloblasts of rats. Arch. Oral Biol. 18: 445-448.

10. RisNes, S. (1979). A method of calculating the speed of movement of ameloblasts during rat incisor amelogenesis. Arch. Oral Biol. 24: 299-306.

11. ROHRSCHNEIDER, L.R. (1980). Adhesion plaques of Rous sarcoma virus-transformed cells contain the src gene product. Proc. Natl. Acad. Sci. USA 77: 3514-3518.

12. SASAKI, T. (1984). Tracer, cytochemical and freeze-fracture study on the mechanisms whereby secretory ameloblasts absorb exogenous proteins. Acta Anat. 118: 23-33.

13. Tsukita, S., Hieda, Y., and Tsukita, S. (1989). A new 82$\mathrm{kD}$ barbed end-capping protein (radixin) localized in the cell-tocell adherens junction: Purification and characterization. J. Cell Biol. 108: 2369-2382.

14. Tsukita, S. and Tsukita, S. (1989). Isolation of cell-to-cell adherens junctions from rat liver. $J$. Cell Biol. 108: 31-41.

15. VolK, T. and GeIGer, B. (1986). A-CAM: a 135-kD receptor of interecellular adherens junctions. I. Immunoelectron microscopic localization and biochemical studies. J. Cell Biol. 103: 1441-1450.

16. VolK, T. and GeIGer, B. (1986). A-CAM: a 135-kD receptor of intercellular adherens junctions. II. Antibody-mediated modulation of junction formation. J. Cell Biol. 103: 14511464.

17. WARShaWSKY, H. (1978). A freeze-fracture study of the topographic relationship between inner enamel-secretory ameloblasts in the rat incisor. Am. J. Anat. 152: 153-203.

18. WARShawsky, H. and SMith, C.E. (1971). a three-dimensional reconstruction of the rods in rat maxillary incisor enamel. Anat. Rec. 169: 585-592.

19. WARShawsKY, H. and Smith, C.E. (1974). Morphological classification of rat incisor ameloblasts. Anat. Rec. 179: 423446.

(Received for publication, March 27, 1990

and in revised form, July 30, 1990) 